\title{
Role of (-)-epigallocatechin-3-gallate in the osteogenic differentiation of human bone marrow mesenchymal stem cells: An enhancer or an inducer?
}

\author{
PAN JIN ${ }^{1,2 *}$, MUYAN LI ${ }^{3 *}$, GUOJIE XU ${ }^{2,4}, \mathrm{KUN} Z \mathrm{ZHANG}^{2,4}, \mathrm{LI} \mathrm{ZHENG}^{2,3}$ and JINMIN ZHAO ${ }^{2,4}$ \\ ${ }^{1}$ Department of Orthopedic Surgery, The First Hospital Affiliated to Henan University, Kaifeng, Henan 475001; \\ ${ }^{2}$ Guangxi Key Laboratory of Regenerative Medicine; ${ }^{3}$ Medical and Scientific Research Center, \\ Guangxi Medical University; ${ }^{4}$ Department of Orthopedic Trauma and Hand Surgery, \\ The First Affiliated Hospital of Guangxi Medical University, \\ Nanning, Guangxi 530021, P.R. China
}

Received October 14, 2014; Accepted May 29, 2015

DOI: $10.3892 /$ etm.2015.2579

\begin{abstract}
Epidemiological investigations have revealed that the consumption of green tea, which is a rich source of (-)-epigallocatechin-3-gallate (EGCG), is associated with a reduced risk of osteoporosis. A number of in vitro and in vivo studies have also demonstrated that EGCG exerts a significant positive effect on osteogenesis; however, the single effect of EGCG on osteogenic differentiation has been seldom studied. EGCG was hypothesized to function as an enhancer or an inducer. In the present study, the effect of EGCG on the osteogenic differentiation of primary human bone marrow mesenchymal stem cells (hBMSCs), without other additives, was investigated. Three groups of stem cells were analyzed, which included a negative control group (hBMSCs cultured with culture medium only), an experimental group (cells treated with culture medium containing 2.5, 5 and $10 \mu \mathrm{M}$ EGCG), and a positive control group (cells cultured with osteogenesis-induced culture medium). After 3, 7, 14 and 21 days, cell proliferation, alkaline phosphatase (ALP) activity and the expression of associated osteogenic genes were analyzed. The results revealed that ALP activity and the expression of associated osteogenic genes, with the exception of bone morphogenetic protein 2 (BMP2), were
\end{abstract}

Correspondence to: Professor Li Zheng, Guangxi Key Laboratory of Regenerative Medicine, Guangxi Medical University, 22 Shuangyong Road, Nanning, Guangxi 530021, P.R. China E-mail: zhengli224@163.com

Professor Jinmin Zhao, Department of Orthopedic Trauma and Hand Surgery, The First Affiliated Hospital of Guangxi Medical University, 22 Shuangyong Road, Nanning, Guangxi 530021, P.R. China

E-mail: zhaojinmin@126.com

*Contributed equally

Key words: epigallocatechin-3-gallate, human bone marrow mesenchymal stem cells, enhancer, inducer, osteogenesis not affected by EGCG treatment alone. These results indicated that EGCG itself had little effect on the osteogenic differentiation of MSCs; however, EGCG was able to enhance osteogenesis in the presence of osteoinductive agents through the upregulation of BMP2 expression. Additionally, EGCG was shown to promote cell growth, demonstrating its safety as a therapeutic agent. Therefore, the present study indicated that treatment with EGCG was dependent on other osteogenic inducers.

\section{Introduction}

A positive correlation between tea consumption and body bone mineral density among postmenopausal females has been reported in numerous countries, such as Canada (1), the UK (2), Taiwan (3), the USA (4), Japan (5) and Australia (6), which may evolve the study of the treatment of osteoporosis. Further investigations have verified that habitual tea consumption contributes to a lower risk of hip fracture $(7,8)$. As the most abundant catechin, which constitutes almost $60 \%$ of the catechins in tea (9), (-)-epigallocatechin-3-gallate (EGCG) has received widespread attention. EGCG is considered to be pharmacologically active (10-12), and play a role in preventing various diseases, including osteoporosis.

EGCG has been reported to protect against bone loss to prevent further deterioration of the bone microarchitecture in ovariectomized or non-ovariectomized rats (13-16). In addition, EGCG has been demonstrated to improve the bone microstructure and quality in aged, orchidectomized rats (17). Rodriguez et al (18) found that a combination of $\alpha$-tricalcium phosphate particles and EGCG effectively stimulated bone regeneration. Furthermore, in vitro tests revealed that EGCG increased the formation of mineralized bone nodules by human osteoblast-like cells (19). Chen et al (20) indicated that EGCG ranging between 1 and $10 \mu \mathrm{M}$ exerted a stimulatory effect on the osteogenesis of murine bone marrow mesenchymal stem cell (MSC) line, D1, by detecting the mRNA expression levels of associated osteogenic genes. By contrast, EGCG has also been shown to induce mouse osteoclast-like cell apoptosis (21) and inhibit the formation of osteoclasts (22). A previous study (23) 
indicated that EGCG may be used as a pro-osteogenic agent for stem cell-based therapy in the treatment of osteoporosis, since EGCG promoted the osteogenic differentiation of primary human bone marrow MSCs (hBMSCs). Thus, EGCG was hypothesized to have the potential to promote osteogenesis. However, the effect of EGCG alone on triggering the onset of osteogenesis in MSCs has seldom been studied. In the majority of cases, osteoinductive agents, such as osteoinductive media or bioactive ceramics, have also been added to stimulate osteogenesis. However, whether EGCG plays the role as an enhancer or an inducer is yet to be answered.

In the present study, the individual effect of EGCG on the osteogenic differentiation of primary hBMSCs, without other additives, was investigated through examining cell proliferation, alkaline phosphatase (ALP) activity and the expression of associated osteogenic genes.

\section{Materials and methods}

Isolation and culture of primary hBMSCs. hBMSCs were harvested from bone marrow extracts obtained from a routine iliac bone graft procedure for the reconstruction of bone defects following a traumatic tibial fracture. The study was approved by the local Ethics Committee of Guangxi Medical University (Nanning, China), and written informed consent was obtained from the patient. The patient was healthy with no specific metabolic or inherited diseases. The bone marrow tissue was flushed out with culture medium of $\alpha$-modified Eagle's medium ( $\alpha$-MEM; Gibco Life Technologies, Carlsbad, CA, USA), supplemented with $10 \%$ (v/v) fetal bovine serum (FBS; Hyclone; GE Healthcare Life Sciences, Logan, UT, USA) and $1 \%(\mathrm{v} / \mathrm{v})$ antibiotics (100 U/1 penicillin and $100 \mathrm{U} / 1$ streptomycin; Solarbio Science \& Technology Co. Ltd., Beijing, China). Following centrifugation (5 min at room temperature, $100 \mathrm{xg}$ ), the cells were suspended in culture medium and maintained in a humidified atmosphere with $5 \%$ $\mathrm{CO}_{2}$ at $37^{\circ} \mathrm{C}$. Non-adherent cells were removed by changing the culture medium every 3 days. When $80-90 \%$ confluence was achieved following 7-10 days of culture, the primary cells were ready for use in the subsequent experiments. The culture medium was changed every 2-3 days.

EGCG treatment. EGCG, with a purity of $\geq 98 \%$ as determined by high performance liquid chromatography, was purchased from Shanghai Yuanye Biotechnology Co. Ltd. (Shanghai, China) and stored at $4^{\circ} \mathrm{C}$. Prior to the experiments, EGCG was dissolved in dimethyl sulfoxide (DMSO; Solarbio Science \& Technology Co. Ltd.) at a concentration of $10 \mathrm{mM}$ and stored at $-20^{\circ} \mathrm{C}$ ready for use. The EGCG stock was diluted with culture medium immediately prior to treatment. Cells were treated with EGCG at various concentrations: $0 \mu \mathrm{M}$ as a negative control and $2.5,5$ and $10 \mu \mathrm{M}$ as experimental groups. The concentration of DMSO was $<0.1 \%$ in all the experiments.

Cell proliferation assay. Effects of EGCG on cell proliferation were assessed using the 3-(4,5-dimethylthiazol-2-y1)-3,5-diphenyltetrazolium bromide (MTT; Sigma-Aldrich, St. Louis, MO, USA) method. Cells were digested with $0.25 \%$ trypsin/EDTA (Sigma-Aldrich), concentrated by centrifuga- tion at $100 \mathrm{x} \mathrm{g}$ for $5 \mathrm{~min}$, resuspended in culture medium and subsequently seeded into 24 -well plates at a density of $5 \times 10^{3}$ cells/well. After $24 \mathrm{~h}$ of culture, the culture medium was replaced with: i) Pure culture medium in the negative control; ii) culture medium with EGCG at concentrations of $2.5,5$ and $10 \mu \mathrm{M}$ in the experimental groups; and iii) osteogenesis-induced culture medium ( $\alpha$-MEM containing $10 \%$ FBS, $50 \mu \mathrm{g} / \mathrm{ml} \mathrm{L}$-ascorbic acid, $10 \mathrm{mM} \beta$-glycerophosphate and $100 \mathrm{nM}$ dexamethasone; Sigma-Aldrich) in the positive control. At days 3, 7, 14 and 21, a solution of MTT in phosphate-buffered saline (PBS; Sigma-Aldrich) was added to each well to a final concentration of $5 \mathrm{mg} / \mathrm{ml}$. Following incubation in a $5 \% \mathrm{CO}_{2}$ incubator (Forma ${ }^{\mathrm{TM}}$ Series II 3110 Water-Jacketed; Thermo Fisher Scientific, Waltham, MA, USA) at $37^{\circ} \mathrm{C}$ for $4 \mathrm{~h}$, the supernatant was discarded and $1 \mathrm{ml}$ DMSO was added to dissolve the formazan crystals. Following thorough and even mixing, samples of $200 \mu \mathrm{l}$ were randomly extracted from each of three parallel wells with the same culture medium three times and transferred to 96-well plates; thus, all samples were performed in nonuplicate. The absorbance value was measured at $570 \mathrm{~nm}$ with a microplate reader (Multiskan ${ }^{\mathrm{TM}}$ GO Microplate Spectrophotometer; Thermo Fisher Scientific, Vantaa, Finland), and the results are shown as units of optical density absorbance values.

ALP activity assay. To examine the ALP activity of the hBMSCs, cells were seeded into 24 -well plates at a density of $5 \times 10^{3}$ cells/well with the different culture media. Following $3,7,14$ and 21 days of culture, the cells were washed with PBS and lysed with $200 \mu 1$ radioimmunoprecipitation assay lysis buffer (Beyotime Institute of Biotechnology, Shanghai, China), which was added to phenylmethanesulfonyl fluoride to form a final concentration of $1 \mathrm{mM}$ prior to the analysis of ALP activity. The total protein concentration $(\mathrm{mg} / \mathrm{ml})$ and ALP activity levels (U/ml) were measured with an enhanced bicinchoninic acid protein assay kit (Beyotime Institute of Biotechnology) and an ALP reagent kit (Nanjing Jiancheng Bioengineering Research Institute, Nanjing, China), respectively, according to the manufacturer's instructions. ALP levels were normalized against the total protein content. All samples were examined in triplicate.

Reverse transcription quantitative polymerase chain reaction (RT-qPCR) assay. RT-qPCR analysis was performed for the detection of osteogenic gene expression levels in the cells cultured in six-well plates from the three groups. Total RNA was extracted with an additional purification step employing an RNA isolation kit (Tiangen Biotech Co. Ltd., Beijing, China) according to the manufacturer's instructions, on days 3, 7, 14 and 21. Subsequently, $1 \mathrm{mg}$ total RNA was reverse transcribed into cDNA. Finally, an ABI 7300 Sequence Detection System (Applied Biosystems Life Technologies, Foster City, CA, USA) was used to conduct RT-qPCR with TaqMan Universal PCR Master Mix and gene-specific TaqMan PCR primers (Applied Biosystems Life Technologies), which included those for bone morphogenetic protein 2 (BMP2), runt-related transcription factor 2 (RUNX2), ALP, bone sialoprotein (BSP), osteocalcin (OCN), $\alpha-1$ type I collagen (COL1A1) and glyceraldehyde-3-phosphate dehydrogenase (GAPDH). Gene expression levels were normalized against those of GAPDH 
Table I. Primers used for reverse transcription quantitative polymerase chain reaction.

\begin{tabular}{llc}
\hline Gene name & \multicolumn{1}{c}{ Forward primer } & \multicolumn{1}{c}{ Reverse primer } \\
\hline GAPDH & 5'-CTATAAATTGAGCCCGCAGC-3' & 5'-GACCAAATCCGTTGACTCCG-3' \\
BMP2 & 5'-TCCATGTGGACGCTCTTTCA-3' & 5'-AGCAGCAACGCTAGAAGACA-3' \\
RUNX2 & 5'-TGTCATGGCGGGTAACGATG-3' & 5'-CCCTAAATCACTGAGGCGGT-3' \\
ALP & 5'-CCAGGGCTGTAAGGACATCG-3' & 5'-GCTCTTCCAGGTGTCAACGA-3' \\
BSP & 5'-CAATCTGTGCCACTCACTGC-3' & 5'-TGCCCTGAACTGGAAATCGTT-3' \\
OCN & 5'-ACACTCCTCGCCCTATTGGC-3' & 5'-CCATTGATACAGGTAGCGCCT-3' \\
COL1A1 & 5'-GTTCAGCTTTGTGGACCTCCG-3' & 5'-GCAGTTCTTGGTCTCGTCAC-3'
\end{tabular}

GAPDH, glyceraldehyde-3-phosphate dehydrogenase; BMP2, bone morphogenetic protein 2; RUNX2, runt-related transcription factor 2; ALP, alkaline phosphatase; BSP, bone sialoprotein; OCN, osteocalcin; COL1A1, $\alpha-1$ type I collagen.

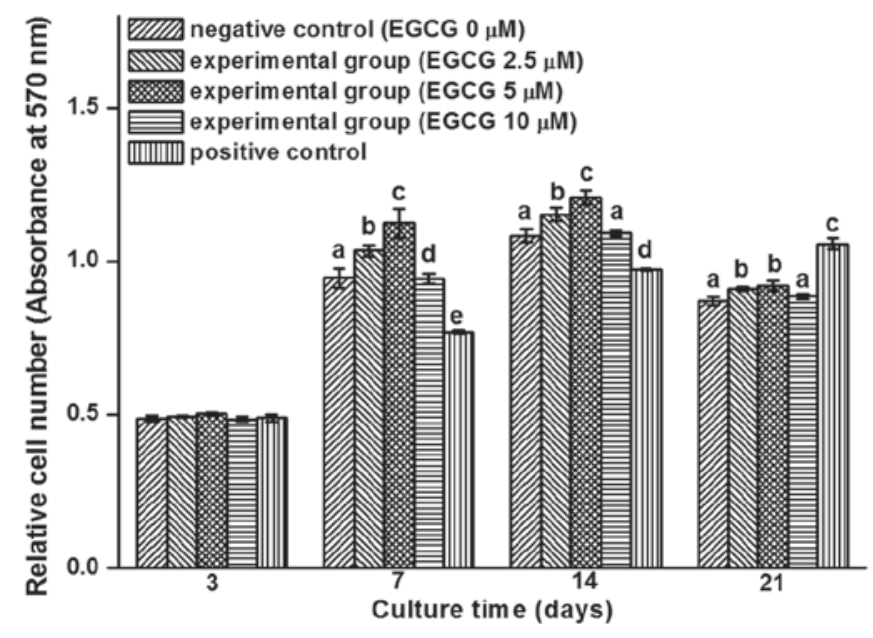

Figure 1. 3-(4,5-dimethylthiazol-2-yl)-2,5-diphenyltetrazolium bromide analysis of cell proliferation in the human bone marrow mesenchymal stem cells (hBMSCs) of the three groups (negative control, hBMSCs were cultured with pure culture medium; experimental groups, cells were treated with culture medium containing 2.5, 5 and $10 \mu \mathrm{M}$ EGCG; positive control, cells were cultured with osteogenesis-induced culture medium). Cell proliferation in the experimental groups was higher compared with the negative control group, while cell proliferation in the positive control group increased over time. In the experimental groups, cell proliferation with $5 \mu \mathrm{M}$ EGCG was higher compared with that of 2.5 and $10 \mu \mathrm{M}$. The bars with different letters at the same time point are significantly different from each other $(P<0.05 ; n=9)$, and those with the same letter exhibit no statistically significant difference. EGCG, (-)-epigallocatechin-3-gallate.

using the comparative $2^{-\Delta \Delta \mathrm{Ct}}$ method. The primers used in this experiment are shown in Table I. All PCR assays were conducted in triplicate.

Immunohistochemical assay. After 7,14 and 21 days of culture, immunohistochemical staining for BMP2 was performed. Cells on the coverslips were washed with PBS, rinsed with $0.01 \%$ Triton X-100 (Beyotime Institute of Biotechnology), washed thoroughly with PBS, treated with $3 \%$ hydrogen peroxide (Wuhan Boster Biological Technology Co. Ltd., Wuhan, China), washed again in PBS and blocked with $3 \%$ bovine serum albumin. Following incubation with a primary antibody against BMP2 (rabbit, anti-human; cat. no. BA0585-1; Wuhan Boster Biological Technology, Ltd.) at a dilution of 1:200, a secondary antibody (goat, anti-rabbit IgG; cat. no. BA1055)

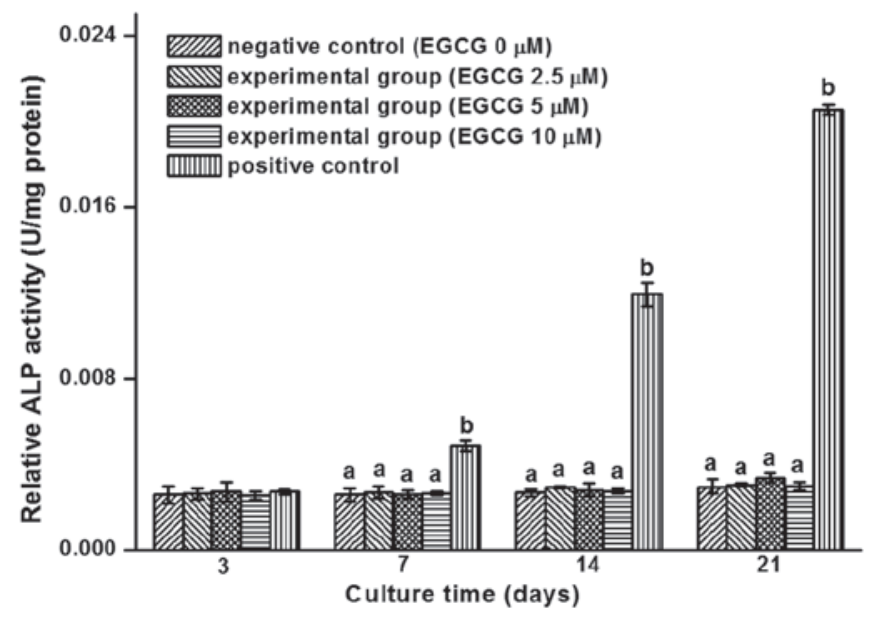

Figure 2. Relative ALP activity (U/mg protein) in the three groups (negative control, human bone marrow mesenchymal stem cells were cultured with pure culture medium; experimental group, cells were treated with culture medium containing 2.5, 5 and $10 \mu \mathrm{M}$ EGCG; positive control, cells were cultured with osteogenesis-induced culture medium). Data are expressed as the mean \pm 2 standard deviations. When compared with the negative control group, the ALP activity in the experimental groups was similar. By contrast, ALP activity in the positive group was significantly higher, becoming increasingly significant with elapsed time. The bars with different letters at the same time point are significantly different from each other $(\mathrm{P}<0.05 ; \mathrm{n}=3)$ and those with the same letter exhibit no statistically significant difference. ALP, alkaline phosphatase; EGCG, (-)-epigallocatechin-3-gallate.

and biotin-labeled horseradish peroxidase were added to the cells. Following incubation with 3,3'-diaminobenzidine and counterstaining with hematoxylin, the cells were air-dried and sealed with neutral resin. The cells were subsequently examined and images were captured using inverted phase contrast microscopy (IX71-F22PH; Olympus Corporation, Tokyo, Japan).

Statistical analysis. Data are presented as the mean \pm 2 standard deviation. All data were evaluated by one-way analysis of variance, whilst the least significance difference multiple comparisons test was performed for further evaluation of the data. The software used was SPSS (version 16.0; SPSS Inc., Chicago, IL, USA). $\mathrm{P}<0.05$ was considered to indicate a statistically significant difference. 
A

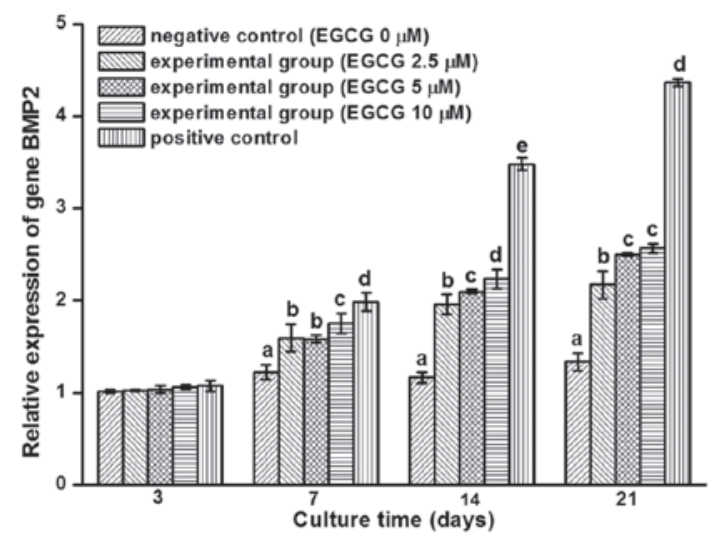

C

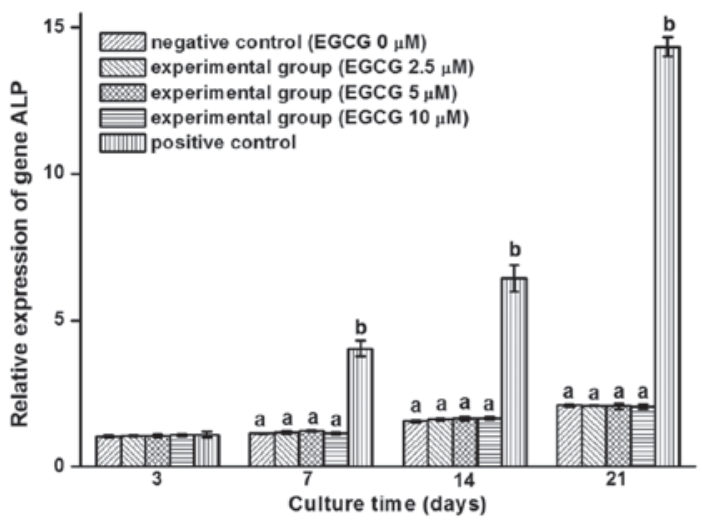

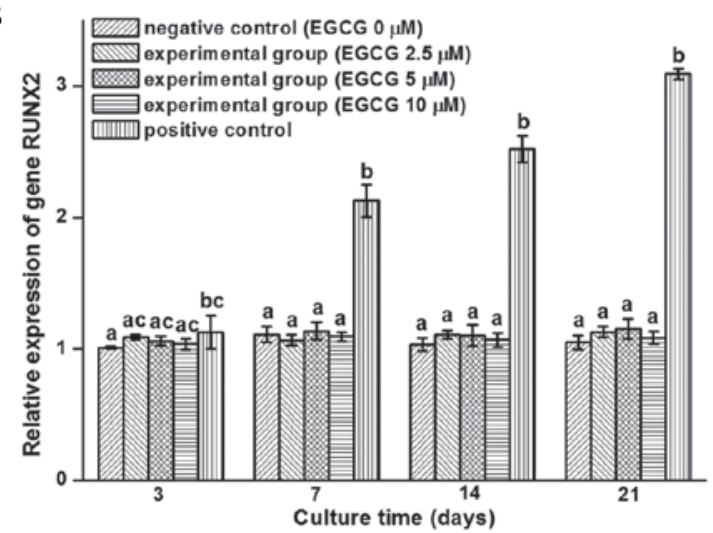

D

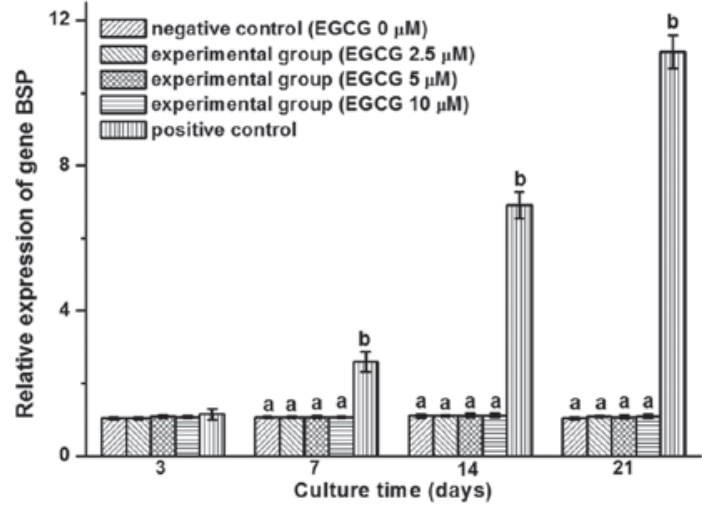

$\mathbf{E}$

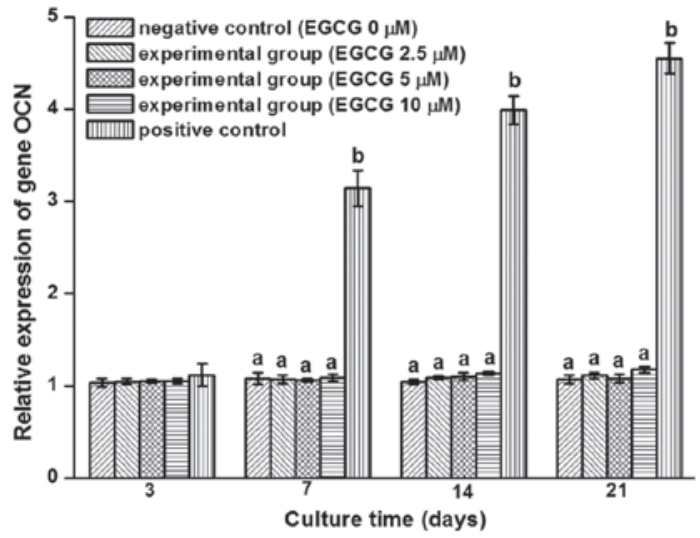

F

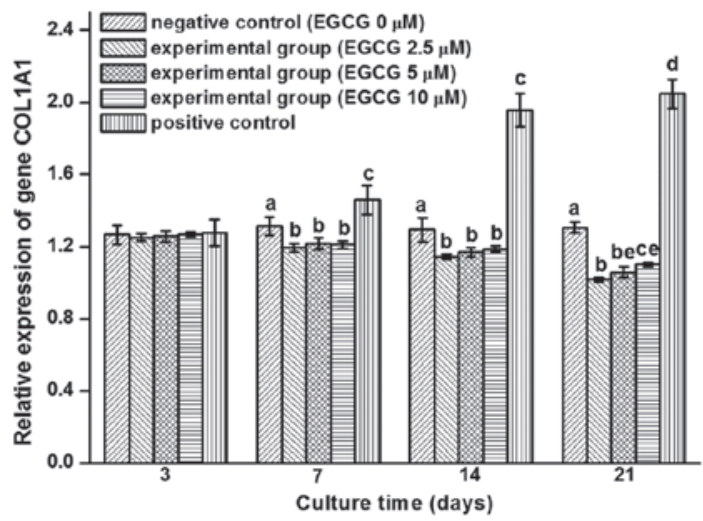

Figure 3. Reverse transcription quantitative polymerase chain reaction was used to analyze the expression of (A) BMP2, (B) RUNX2, (C) ALP, (D) BSP, (E) OCN and (F) COL1A1 osteogenic genes in human bone marrow mesenchymal stem cells (hBMSCs) cultured in the different groups for 3, 7 , 14 and 21 days (negative control, hBMSCs cultured with pure culture medium; experimental, cells treated with culture medium containing 2.5, 5 and $10 \mu$ M EGCG; positive control, cells cultured with osteogenesis-induced culture medium). In the positive control group, all the detected genes were upregulated over time. When compared with the negative control group, the expression of the genes showed no change, with the exception of the BMP2 gene in the experimental group that was upregulated, and the expression of the COL1A1 gene, which exhibited a downward trend with time. The minimum value was set to 1 , and values are expressed as the mean \pm 2 standard deviations. The bars with different letters at the same time point are significantly different from each other $(\mathrm{P}<0.05 ; \mathrm{n}=3$ ), and those with the same letter exhibit no statistically significant difference. BMP2, bone morphogenetic protein 2; RUNX2, runt-related transcription factor 2; ALP, alkaline phosphatase; BSP, bone sialoprotein; OCN, osteocalcin; COL1A1, $\alpha$-1 type I collagen; EGCG, (-)-epigallocatechin-3-gallate.

\section{Results}

Cell proliferation assay. The effect of EGCG on the proliferation of hBMSCs was examined by an MTT assay. hBMSCs were treated with different concentrations of EGCG $(0 \mu \mathrm{M}$ in the negative control group, and $2.5,5$ and $10 \mu \mathrm{M}$ in the experimental groups) or osteogenic-induced culture medium in the positive control group. As shown in Fig. 1, the rate of cell proliferation at day 3 following treatment with EGCG in the range of $0-10 \mu \mathrm{M}$ was comparable with that in the positive control group; therefore, the drug was demonstrated to be non-toxic to the cells, which was in agreement with the previous (pilot) study (23). Although no statistically significant difference was observed between the groups on day 3 , cell proliferation was evidently promoted by $5 \mu \mathrm{M}$ EGCG between days 7 and 14. On day 21, the number of cells in the positive control group 

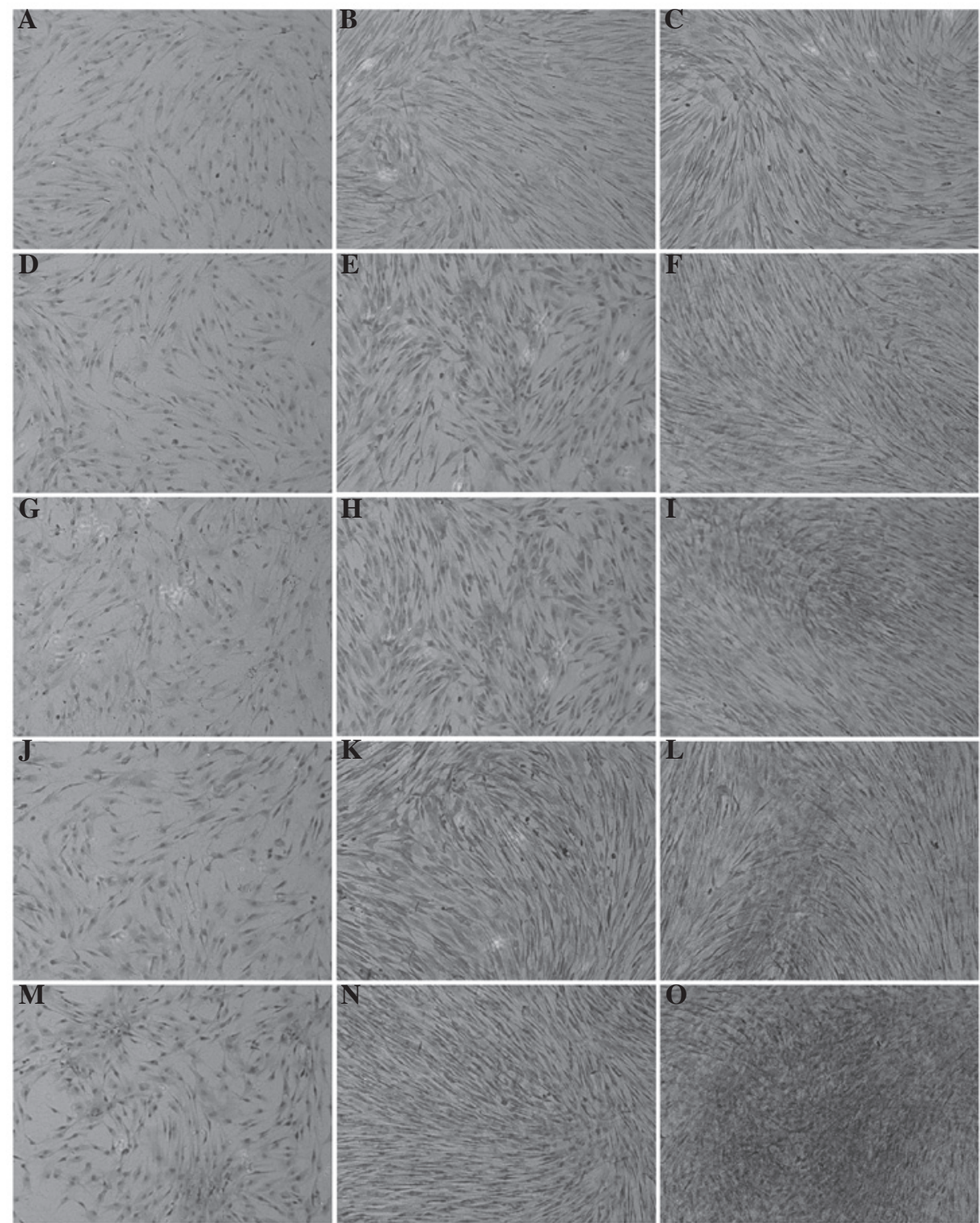

Figure 4. Immunohistochemical staining of bone morphogenetic protein 2 expression in human bone marrow mesenchymal stem cells (hBMSCs) of the three groups [negative control, hBMSCs cultured with pure culture medium; experimental, cells treated with culture medium containing $2.5,5$ and $10 \mu \mathrm{M}$ (-)-epigallocatechin-3-gallate (EGCG); positive control, cells cultured with osteogenesis-induced culture medium]. (A-C) Staining of hBMSCs in the negative control group on days 7, 14 and 21, respectively. (D-F) Staining of hBMSCs in the $2.5 \mu \mathrm{M}$ EGCG experimental group on days 7, 14 and 21, respectively. (G-I) Staining of hBMSCs in the $5 \mu \mathrm{M}$ EGCG experimental group on days 7, 14 and 21, respectively. (J-L) Staining of hBMSCs in the $10 \mu \mathrm{M}$ EGCG experimental group on days 7, 14 and 21, respectively. (M-O) Staining of hBMSCs in the positive control group on days 7, 14 and 21, respectively. The staining in the positive control group was evidently greater, while that in the other groups was dose-dependently upregulated.Magnification, x100.

was significantly higher compared with the other groups, which may be attributed to the limit of the cell culture area (Fig. 1). Therefore, the results indicated that EGCG facilitated the growth of hBMSCs, particularly at a concentration of $5 \mu \mathrm{M}$.

ALP activity assay. ALP is produced by osteoblasts and is hypothesized to be involved in the degradation of inorganic pyrophosphates to provide sufficient local phosphate or inorganic pyrophosphate for the occurrence of mineralization (24). Commonly used as a marker of osteogenesis, ALP activity is considered to reflect the degree of osteogenic differentiation.

An ALP activity assay was used for the quantitative assessment of the units of activity per mg protein in the three groups, namely the negative control (hBMSCs cultured with pure culture medium), experimental groups (cells treated with culture medium containing $2.5,5$ and $10 \mu \mathrm{M}$ EGCG respectively) and positive control (cells cultured with osteogenesis-induced culture medium). The histogram in Fig. 2 shows that the ALP activity levels primarily remained unchanged with increasing concentrations of EGCG; however, the activity level increased significantly in the positive control over time.

RT-qPCR assay. The effect of EGCG on hBMSCs in the three groups (negative control, experimental, positive control) was further examined through gene expression analysis of BMP2, RUNX2, ALP, BSP, OCN and COL1A1 following 3, 7, 14 and 21 days of culture. Expression of the BMP2 gene was dose-dependently upregulated; however, the expression 
levels of the other genes exhibited no response to the different doses of EGCG, and the expression of the COL1A1 gene following treatment with EGCG had a tendency to be inferior to the controls. As a positive control, the expression of all the detected genes in the positive group were superior to the other groups (Fig. 3).

Immunohistochemical staining. Further investigation into the expression of osteogenesis-associated proteins was performed using immunocytochemistry with a specific antibody for BMP2 (Fig. 4). BMP2 is one of the members of the transforming growth factor- $\beta$ superfamily. The primary function of BMP2 is the promotion of osteogenic differentiation (25-30). As shown in Fig. 4, EGCG clearly upregulated the protein expression levels of BMP2, with stronger positive staining observed when compared with the control, which is consistent with the RT-qPCR results. The staining results indicated that the protein expression of BMP2 increased over time.

\section{Discussion}

EGCG, as the most abundant catechin and a major bioactive component of green tea (31), has been reported by a number of studies (19-23) to have an association with osteogenesis. However, the association is dependent on an osteogenic inducer, such as inductive media.

In the present study, the individual effect of EGCG on osteogenesis was analyzed to clarify the role of EGCG in the function of MSC differentiation. The results revealed that EGCG had little effect on the osteogenic differentiation of MSCs, as evidenced by only a marginal influence on ALP activity (Fig. 2) and the expression of osteogenic genes, including RUNX2, ALP, BSP, OCN and COL1A1 (Fig. 3B-F). In accordance with the present study, EGCG has a positive effect on proliferation and little effect on the osteogenic differentiation without additional osteoinductive agents. Kamon et al (32) also demonstrated that EGCG exerted a suppressive effect on the differentiation of precursor cells of osteoblasts, although EGCG had little effect on differentiated osteoblasts. However, certain studies (20) have shown that EGCG can promote the osteogenic differentiation of osteoblast precursors. These observations indicate that EGCG functions more as an enhancer rather than an inducer in osteogenic differentiation.

Notably, EGCG treatment was shown to upregulate BMP2 expression in a dose-dependent manner (Figs. 3A and 4). EGCG has been hypothesized to induce osteogenesis at a relatively high concentration, but within the recommended amount, which is more than the physiological level and less than the detrimental level $(33,34)$. However, EGCG treatment alone is unable to produce sufficient levels of BMP2 to trigger osteogenesis. By contrast, EGCG can improve the facilitation of osteogenesis in the presence of osteoinductive agents through coregulation of the BMP/Smad signaling pathways (35-37). Thus, the effect of EGCG on osteogenesis relies on the presence of an osteogenic inducer.

EGCG alone has a proliferative effect on the proliferation of hMSCs. In particular, EGCG was shown to evidently promote cell proliferation at a concentration of $5 \mu \mathrm{M}$. Furthermore, a previous (pilot) study (23) demonstrated that
EGCG supports cell proliferation in the presence of osteoinductive media. The study by Yagi et al (34) lent potent support for the present study by elucidating that EGCG is capable of suppressing inducible oxidative stress that may comprise cellular processes, including cell proliferation. Therefore, EGCG is biocompatible and the results support the possibility of clinical use.

In conclusion, EGCG alone has little effect on the osteogenic differentiation of MSCs, and the catechin only plays a role when combined with osteoinductive agents through the upregulation of BMP2 expression. In addition, EGCG is able to sustain cell proliferation, indicating the safety of the compound for clinical application. Therefore, the present study indicated that further development of EGCG as a pro-osteogenic agent should be coupled with additional osteoinductive agents.

\section{Acknowledgements}

This study was financially supported by the National Science and Technology Pillar Program of China (grant no. 2012BAI42G00), Guangxi Scientific Research and Technological Development Foundation (grant no. Guikehe 14125008-2-14) and Guangxi Science Fund for Distinguished Young Scholars (grant no. 2014GXNSFGA118006). This study has also been supported by the Research Center for Regenerative Medicine of Guangxi Medical University and Collaborative Innovation Center of Guangxi Biological Medicine.

\section{References}

1. Hoover PA, Webber CE, Beaumont LF and Blake JM: Postmenopausal bone mineral density: Relationship to calcium intake, calcium absorption, residual estrogen, body composition and physical activity. Can J Physiol Pharmacol 74: 911-917, 1996.

2. Hegarty VM, May HM and Khaw KT: Tea drinking and bone mineral density in older women. Am J Clin Nutr 71: 1003-1007, 2000.

3. Wu CH, Yang YC, Yao WJ, et al: Epidemiological evidence of increased bone mineral density in habitual tea drinkers. Arch Intern Med 162: 1001-1006, 2002.

4. Chen Z, Pettinger MB, Ritenbaugh C, et al: Habitual tea consumption and risk of osteoporosis: A prospective study in the women's health initiative observational cohort. Am J Epidemiol 158: 772-781, 2003.

5. Muraki S, Yamamoto S, Ishibashi H, et al: Diet and lifestyle associated with increased bone mineral density: Cross-sectional study of Japanese elderly women at an osteoporosis outpatient clinic. J Orthop Sci 12: 317-320, 2007.

6. Devine A, Hodgson JM, Dick IM and Prince RL: Tea drinking is associated with benefits on bone density in older women. Am J Clin Nutr 86: 1243-1247, 2007.

7. Johnell O, Gullberg B, Kanis JA, et al: Risk factors for hip fracture in European women: The MEDOS study. Mediterranean Osteoporosis Study. J Bone Miner Res 10: 1802-1815, 1995.

8. Kanis J, Johnell O, Gullberg B, et al: Risk factors for hip fracture in men from southern Europe: The MEDOS study. Mediterranean Osteoporosis Study. Osteoporos Int 9: 45-54, 1999.

9. Harbowy ME and Balentine DA: Tea chemistry. Crit Rev Plant Sci 16: 415-480, 1997

10. Chen $\mathrm{CH}$, Ho ML, Chang JK, et al: Green tea catechins enhance the expression of osteoprotegerin (OPG) in pluripotent stem cells. J Orthop Surg Taiwan 20: 178-183, 2003.

11. Dulloo AG, Duret C, Rohrer D, et al: Efficacy of a green tea extract rich in catechin polyphenols and caffeine in increasing 24-h energy expenditure and fat oxidation in humans. Am J Clin Nutr 70: 1040-1045, 1999.

12. Stagg GV and Millin DJ: The nutritional and therapeutic value of tea - a review. J Sci Food Agric 26: 1439-1459, 1975 
13. Shen CL, Wang P, Guerrieri J, et al: Protective effect of green tea polyphenols on bone loss in middle-aged female rats. Osteoporos Int 19: 979-990, 2008.

14. Shen CL, Yeh JK, Stoecker BJ, et al: Green tea polyphenols mitigate deterioration of bone microarchitecture in middle-aged female rats. Bone 44: 684-690, 2009.

15. Shen CL, Yeh JK, Cao JJ, et al: Green tea polyphenols mitigate bone loss of female rats in a chronic inflammation-induced bone loss model. J Nutr Biochem 21: 968-974, 2010.

16. Shen CL, Yeh JK, Samathanam C, et al: Green tea polyphenols attenuate deterioration of bone microarchitecture in female rats with systemic chronic inflammation. Osteoporos Int 22: 327-337, 2011.

17. Shen CL, Cao JJ, Dagda RY, et al: Supplementation with green tea polyphenols improves bone microstructure and quality in aged, orchidectomized rats. Calcif Tissue Int 88: 455-463, 2011.

18. Rodriguez R, Kondo H, Nyan M, et al: Implantation of green tea catechin $\alpha$-tricalcium phosphate combination enhances bone repair in rat skull defects. J Biomed Mater Res B Appl Biomater 98: 263-271, 2011.

19. Vali B, Rao LG and El-Sohemy A: Epigallocatechin-3-gallate increases the formation of mineralized bone nodules by human osteoblast-like cells. J Nutr Biochem 18: 341-347, 2007.

20. Chen CH, Ho ML, Chang JK, et al: Green tea catechin enhances osteogenesis in a bone marrow mesenchymal stem cell line. Osteoporos Int 16: 2039-2045, 2005.

21. Nakagawa $\mathrm{H}$, Wachi M, Woo JT, et al: Fenton reaction is primarily involved in a mechanism of (-)-epigallocatechin-3-gallate to induce osteoclastic cell death. Biochem Bioph Res Commun 292: 94-101, 2002.

22. Yun JH, Pang EK, Kim CS, et al: Inhibitory effects of green tea polyphenol (-)-epigallocatechin gallate on the expression of matrix metalloproteinase- 9 and on the formation of osteoclasts. J Periodontal Res 39: 300-307, 2004.

23. Jin $\mathrm{P}, \mathrm{Wu} \mathrm{H}, \mathrm{Xu} \mathrm{G}$, et al: Epigallocatechin-3-gallate (EGCG) as a pro-osteogenic agent to enhance osteogenic differentiation of mesenchymal stem cells from human bone marrow: An in vitro study. Cell Tissue Res 356: 381-390, 2014.

24. Na K, Sun BK, Woo DG, et al: Osteogenic differentiation of rabbit mesenchymal stem cells in thermo-reversible hydroge constructs containing hydroxyapatite and bone morphogenic protein-2 (BMP-2). Biomaterials 28: 2631-2637, 2007.
25. Harris SE, Bonewald LF, Harris MA, et al: Effects of transforming growth factor beta on bone nodule formation and expression of bone morphogenetic protein 2 , osteocalcin, osteopontin, alkaline phosphatase and type I collagen mRNA in long-term cultures of fetal rat calvarial osteoblasts. J Bone Miner Res 9: 855-863, 1994.

26. Hogan BL: Bone morphogenetic proteins: Multifunctional regulators of vertebrate development. Genes Dev 10: 1580-1594, 1996.

27. Reddi AH: Bone and cartilage differentiation. Curr Opin Genet Dev 4: 737-744, 1994.

28. Wozney JM, Rosen V, Celeste AJ, et al: Novel regulators of bone formation: Molecular clones and activities. Science 242: 1528-1534, 1988 .

29. Wozney JM: The bone morphogenetic protein family and osteogenesis. Mol Reprod Dev 32: 160-167, 1992.

30. Yamaguchi A, Katagiri T, Ikeda T, et al: Recombinant human bone morphogenetic protein-2 stimulates osteoblastic maturation and inhibits myogenic differentiation in vitro. J Cell Biol 113: 681-687, 1991.

31. Harborne JB and Williams CA: Advances in flavonoid research since 1992. Phytochemistry 55: 481-504, 2000.

32. Kamon M, Zhao R and Sakamoto K: Green tea polyphenol (-)-epigallocatechin gallate suppressed the differentiation of murine osteoblastic MC3T3-E1 cells. Cell Biol Int 34: 109-116, 2009.

33. Yang $\mathrm{CH}$, Lin $\mathrm{CY}$, Yang JH, et al: Supplementary catechins attenuate cooking-oil-fumes-induced oxidative stress in rat lung. Chin J Physiol 52: 151-159, 2009.

34. Yagi H, Tan J and Tuan RS: Polyphenols suppress hydrogen peroxide-induced oxidative stress in human bone-marrow derived mesenchymal stem cells. J Cell Biochem 114: 1163-1173, 2013.

35. Hanai J, Chen LF, Kanno T, et al: Interaction and functional cooperation of PEBP2/CBF with Smads. Synergistic induction of the immunoglobulin germline C-alpha promoter. J Biol Chem 274: 31577-31582, 1999

36. Javed A, Barnes GL, Jasanya BO, et al: Runt homology domain transcription factors (Runx, Cbfa and AML) mediate repression of the bone sialoprotein promoter: Evidence for promoter context-dependent activity of Cbfa proteins. Mol Cell Biol 21: 2891-2905, 2001.

37. Nishimura R, Hata K, Harris SE, et al: Core-binding factor alpha 1 (Cbfa1) induces osteoblastic differentiation of $\mathrm{C} 2 \mathrm{C} 12$ cells without interactions with Smad1 and Smad5. Bone 31: 303-312, 2002. 